\title{
Conhecimento sobre hanseníase de contatos intradomiciliares na Atenção Primária em Ananindeua, Pará, Brasil
}

\author{
Knowledge of household contacts of leprosy in the Primary Care in Ananindeua, Pará, Brazil \\ Conocimiento sobre la lepra de contactos intra-domicilares en la Atención Primaria en \\ Ananindeua, Pará, Brasil
}

Lorena Margalho Sousa. Universidade Federal do Pará (UFPA). Iorenasousa36@yahoo.com.br (Autora correspondente)

Lorena Colares Maranhão. Universidade Federal do Pará (UFPA). Iorenaitb@yahoo.com.br

Carla Andréa Avelar Pires. Universidade Federal do Pará (UFPA). carlaavelarpires@bol.com.br

Danielle de Moraes Rodrigues. Universidade Federal do Pará (UFPA). danylegal98@hotmail.com

\section{Resumo}

Este relato buscou identificar o grau de conhecimento de contatos intradomiciliares de pacientes hansenianos tratados e acompanhados em uma Unidade de Saúde da Família (USF) no município de Ananindeua-Pará. Após visitas domiciliares, aplicação de protocolo de pesquisa e exame físico, nenhum caso suspeito de hanseníase foi encontrado, no entanto, o conhecimento sobre a doença ainda é escasso, pois apenas $20 \%$ dos contatos responderam que se tratava de uma "doença de pele com cura". Sobre a representação da doença para os entrevistados, $20 \%$ responderam ter "medo de contrair a doença". Metade da amostra sabia a forma de transmissão e 10\% não souberam emitir nenhuma resposta; $60 \%$ dos contatos responderam que a hanseníase deve ser tratada na unidade de saúde com medicamentos orais. Aqueles que receberam orientações prévias possuíam melhores esclarecimentos sobre a doença (50\%). É preciso um acompanhamento adequado desses contatos, além de uma atuação mais efetiva em educação em saúde.

\section{Abstract}

This experience report sought to identify the degree of knowledge of household contacts of leprosy patients treated and monitored in a Family Health Center in the municipality of Ananindeua, state of Pará. After home visits, application of a research protocol, and physical examination, no suspected case of leprosy was found; however, knowledge about the disease is still scarce, since only $20 \%$ of the contacts answered that leprosy is a "treatable skin disease". Regarding the representation of the disease for the interviewees, $20 \%$ reported having "fear of contracting the disease". Half of the sample were aware of its mode of transmission and $10 \%$ were unable to give any answer; $60 \%$ of the contacts answered that leprosy should be treated at the health center with oral drugs. Those who had received advance directives were better informed about the disease (50\%). A proper follow up of these contacts is needed, as well as a more effective health education.

\section{Resumen}

Este relato de experiencia trató de determinar el grado de conocimiento de los contactos intra-domicilares de enfermos de lepra tratados y seguidos en una Unidad de Salud Familiar (USF) en la ciudad de Ananindeua-Pará. Después de las visitas domicilares, de la aplicación del protocolo de investigación y del examen físico, no fue encontrado ningún caso sospechoso de lepra, sin embargo el conocimiento sobre la enfermedad sigue siendo escaso, ya que sólo el 20\% de los contactos respondió que se trataba de una "enfermedad curable de la piel". Sobre la representación de la enfermedad entre los encuestados, 20\% informó tener "miedo a contraer la enfermedad". La mitad de la muestra conocía el modo de transmisión y el 10\% no pudo dar ninguna respuesta; el $60 \%$ de los contactos respondió que la lepra debe ser tratada en una USF con medicamentos orales. Los que recibieron orientaciones previas tuvieron mayores conocimientos sobre la enfermedad (50\%). Se necesita un acompañamiento adecuado de esos contactos, así como una actuación más efectiva en educación para la salud.
Palavras-chave:

Atenção Primária à Saúde Hanseníase

Unidade Saúde da Família

Keywords:

Primary Health Care

Leprosy

Family Health Centre

\section{Palabras clave:}

Atención Primaria de la Salud Lepra

Unidad de Salud Familiar 


\section{Introdução}

No contexto da construção do Sistema Único de Saúde (SUS), a instituição da Estratégia Saúde da Família (ESF) consiste em um eixo estruturante para organizar a transformação do modelo de atenção à saúde. Nesse sentido, um dos propósitos das equipes da ESF é a educação em saúde, definida como "um campo de práticas que se dá ao nível das relações sociais, normalmente estabelecidas pelos profissionais de saúde entre si e a instituição e com os usuários no desenvolvimento cotidiano de suas atividades"'. Assim, a equipe deve estar apta a prestar assistência integral e contínua às famílias de uma determinada área, identificando situaçôes de risco à saúde na comunidade assistida e em parceria com ela, enfrentando os determinantes do processo saúde-doença e desenvolvendo ações educativas para a melhoria do autocuidado dos indivíduos ${ }^{2}$.

Entre as responsabilidades e açôes estratégicas mínimas que todos os municípios brasileiros devem desenvolver no âmbito da Atenção Primária à Saúde (APS) encontra-se o controle da hanseníase, doença infectocontagiosa causada pelo Mycobacterium leprae, de evolução crônica e que atinge predominantemente a pele e os nervos periféricos do indivíduo. É uma doença potencialmente incapacitante e, embora curável, seu diagnóstico causa grande impacto psicossocial, pelos preconceitos e estigmas que a envolvem ${ }^{3,4}$.

A afecção pode atingir pessoas de todas as idades e sexos. Contudo, o risco de adoecer está ligado a vários fatores, dentre eles nível da endemia e condiçôes socioeconômicas desfavoráveis ${ }^{5}$.

O Brasil é responsável por cerca de $85 \%$ dos casos registrados nas Américas. Atualmente, o país ocupa o segundo lugar em número de doentes, após a Índia ${ }^{3}$ e, apesar da tendência de estabilização dos coeficientes de detecção de hanseníase no país, as regiōes Norte, Centro-Oeste e Nordeste são as que apresentam os mais altos patamares ${ }^{6}$.

A Região Norte é a que apresenta o maior coeficiente de prevalência regional e o Pará é um dos estados que ainda hoje apresentam sérias dificuldades para desenvolver o Programa de Controle da Hanseníase, necessitando intensificar esforços na luta contra o estigma social e na conscientização dos profissionais da área da saúde 7 .

O controle da doença era realizado pela Área Técnica de Dermatologia Sanitária, a qual foi incorporada, em 2000, ao Departamento de Atenção Básica do Ministério da Saúde, com o intuito de utilizar o Programa Saúde da Família (PSF) e o Programa de Agentes Comunitários de Saúde (PACS) para a descentralização do diagnóstico e tratamento da hanseníase ${ }^{8}$.

O comunicante ou contato de um paciente com hanseníase é o indivíduo com maior risco de adquirir a doença e, além disso, possui grande importância na cadeia epidemiológica dessa doença. As atividades relacionadas ao controle dos comunicantes têm sido pouco valorizadas no contexto familiar e social, pois existe um maior direcionamento ao controle da doença e do indivíduo doente ${ }^{9,10}$.

Em busca do cumprimento da meta de controle da hanseníase preconizada pela Organização Mundial da Saúde (OMS) e pretendida pelo Ministério da Saúde até o ano de 2010 tornou-se necessário investir em açóes efetivas para o diagnóstico da doença e na busca e controle dos contatos intradomiciliares para a quebra da cadeia de transmissão ${ }^{11}$.

As atividades relacionadas ao controle da hanseníase, pela equipe da APS, através da busca e controle dos comunicantes são açôes que não podem ser subestimadas, seja em áreas de alta prevalência seja em locais de baixa endemicidade. Dentre essas açôes de controle da doença, a busca da identificação da fonte de infecção do doente previne a continuação do contágio de outras pessoas ${ }^{12}$.

Este relato propóe demonstrar o conhecimento de uma doença infectocontagiosa por parte de contatos intradomiciliares de pacientes hansênicos tratados pela Estratégia Saúde da Família no norte do Pará. Tendo em vista a grande importância dos contatos intradomiciliares que determinam a infecção ou reinfecção da hanseníase, este estudo buscou relatar atividades realizadas por meio de visitas domiciliares por uma equipe multiprofissional voltada à educação para a saúde e à prevenção, averiguando o grau de conhecimento sobre a hanseníase por parte desses comunicantes intradomiciliares. 


\section{Desenvolvimento}

Este estudo integra o projeto de pesquisa intitulado Busca Ativa dos Contatos Intradomiciliares dos Pacientes Hansênicos Matriculados e Tratados na Estratégia Saúde da Família da Cidade Nova VII, no período de Setembro de 2007 a Setembro de 2010, de CAAE 0073.0.073.000-10 e parecer n. 128/10 - CEP-ICS/UFPA.

Trata-se de um estudo descritivo, que relata a experiência de uma equipe da ESF na busca de contatos intradomiciliares de todos os pacientes hansênicos tratados e acompanhados em uma USF no município de Ananindeua, Pará, no período de setembro de 2007 a setembro de 2010, com o intuito de identificar o grau de conhecimento que esses comunicantes detinham sobre a doença que os familiares tiveram e trataram.

Inicialmente foi realizado um levantamento de todos os pacientes tratados nessa unidade de saúde no período citado, depois a atividade seguiu por meio de visitas domiciliares realizadas por uma equipe multiprofissional formada por enfermeira, agentes comunitários de saúde e acadêmicas de Medicina também pertencentes a um projeto do governo federal e de uma universidade pública.

A atividade baseou-se na aplicação de um questionário estruturado elaborado pelos acadêmicos e na realização de exame físico dos contatos intradomiciliares. A análise dos dados foi realizada de forma eletrônica, utilizando-se os programas Microsoft Office Excel 2007 e Microsoft Office Word 2007 e organizando as informaçóes em tabelas e gráficos. Num primeiro momento, nos endereços fornecidos pelos pacientes-índice, foram realizadas as visitas domiciliares para quantificar o número de pessoas que conviviam com os pacientes anteriormente tratados.

Num segundo momento foi aplicado o protocolo, no qual foram coletados dados qualitativos relacionados aos conhecimentos sobre hanseníase, como o conceito de hanseníase, o que a doença representa na vida da pessoa, como ocorre a transmissáo e como e onde é feito o tratamento. Tendo em vista que $40 \%$ dos pacientes-índice apresentavam de 3 a 5 contatos e que o tempo de convivência após o diagnóstico de hanseníase, para $40 \%$ deles, correspondeu a 5 a 10 anos e, para 30\%, a mais de 15 anos, houve a necessidade de realizar, no último momento da pesquisa, o exame físico verificando lesôes suspeitas de relacionarem-se à doença, considerando também que 100\% dos pacientes-índice foram acometidos pelas formas multibacilares da hanseníase. Constatou-se, também, a presença de cicatriz vacinal da BCG em 100\% dos contatos.

A realização desse tipo de pesquisa propiciou aos participantes uma visão integral e holística do paciente, assim como a investigação de possíveis casos, ministrar orientação sobre hanseníase para os contatos intradomiciliares dos doentes e ressaltar para eles a importância do tratamento e sua cura, todas sob supervisão do preceptor.

Posteriormente, os acadêmicos refletiram sobre a experiência, identificaram aspectos positivos e negativos e propuseram sugestôes para a melhoria da atenção prestada à comunidade, consolidando também o processo de ensino-aprendizagem. Além de conhecerem essa doença num contexto local, foi também propiciado a esses acadêmicos a aproximação com a realidade da população investigada, contato com uma prática humanizada e com o trabalho em equipe.

Constatou-se entre os contatos intradomiciliares que, apesar de familiares próximos terem sido acometidos pela hanseníase, o conhecimento sobre a doença ainda era escasso, embora a escolaridade da amostra em grande parte corresponda a ensino médio, incompleto e completo (80\%). Quando indagados sobre o conceito de hanseníase, $20 \%$ dos contatos responderam que se tratava de "uma doença de pele com cura", assim como $20 \%$ relataram ser "uma doença que se pega pelo ar". Outras respostas corresponderam a "doença que se pega pelo contato, ar e fala" e "doença que a pessoa não precisa ficar isolada", correspondendo cada uma a $10 \%$.

Sobre a representação da doença para os entrevistados, $20 \%$ responderam ter "medo de contrair a doença", $10 \%$ responderam que a hanseníase é uma doença que "prejudica o organismo", 10\% citaram que "deixa sequelas" e outros 10\% responderam que é uma doença que "estressa e irrita". Além disso, $10 \%$ responderam que "a pessoa pode morrer se não fizer o tratamento".

A forma de transmissão da hanseníase, para $50 \%$ dos contatos, foi referida como sendo pelo ar/ar e fala/fala; $10 \%$ não tinham nenhuma resposta; $10 \%$ responderam que podia ser transmitida por qualquer tipo de contato, incluindo relaçáo sexual. Perguntados sobre como e onde tratar a hanseníase, $60 \%$ dos contatos responderam que devia ser tratada na USF e que o tratamento é medicamentoso. Notou-se que aqueles que receberam orientaçóes na USF ou pelo profissional de saúde tinham melhor compreensão sobre a doença (50\%). 


\section{Conclusão}

Essa vivência fora do contexto de sala de aula contribuiu para o entendimento das reais necessidades dos usuários e para um maior conhecimento do processo de trabalho no campo da APS envolvendo uma equipe multidisciplinar. A experiência foi percebida pelos estudantes como importante ferramenta na sua formação, pois ampliou seu conceito de saúde, melhorou a sua compreensão do Sistema Único de Saúde e dos seus princípios e os levou a refletir sobre questóes sociais que envolvem a maior parte da população, experiências dificilmente demonstráveis nas aulas ou encontradas no seu cotidiano social.

Apesar de não ter sido encontrado nenhum caso suspeito de hanseníase entre os contatos intradomiciliares, foi constatado que aqueles que receberam orientaçóes pela Unidade de Saúde foram os que detinham maior conhecimento sobre a doença. No entanto, é necessário que a equipe de saúde da referida Unidade faça o acompanhamento adequado desses contatos, devido aos fatores que podem levar ao adoecimento.

É indispensável também reforçar atividades educativas para a população, informando de forma adequada as características da doença e a importância do diagnóstico e do tratamento. A equipe da ESF também deve ser estimulada a exercer de forma mais enfática seu papel em relação à educação em saúde, pois notou-se que aqueles contatos intradomiciliares que detinham maiores conhecimentos eram justamente aqueles que tinham sido orientados pela equipe. Além disso, os que possuíam algum conhecimento apresentavam certas distorçóes, evidenciando falta de esclarecimento, mesmo entre aqueles que tiveram familiares acometidos pela doença. Observou-se também que apesar da hanseníase ser uma doença com grande importância epidemiológica na região Norte, a população possui pouco ou nenhum conhecimento sobre essa doença. É preciso um acompanhamento adequado desses contatos, além de uma atuação mais efetiva em educação em saúde.

\section{Referências}

1. L’Abbate S. Educação em saúde: uma nova abordagem. Cad Saúde Pública. 1994; 4: 481-90. http://dx.doi.org/10.1590/S0102-311X1994000400008

2. Alves VS. Um modelo de educação em saúde para o programa saúde da família: pela integralidade da atenção e reorientação do modelo assistencial. Interface: Comun, Saúde Educ. 2005; 9: 39-52. http://dx.doi.org/10.1590/S1414-32832005000100004

3. Azulay RD, Azulay DR. Dermatologia. Rio de Janeiro: Guanabara Koogan; 2008. p. 322-324.

4. Brasil. Ministério da Saúde. Secretaria de Atenção à Saúde. Departamento de Atenção Básica. Programa Saúde da Família: ampliando a cobertura para consolidar a mudança do modelo de Atenção Básica. Brasília; 2003.

5. Brasil. Ministério da Saúde. Fundação Nacional de Saúde. Guia de Vigilância Epidemiológica. Brasília; 2002.

6. Brasil. Ministério da Saúde. Secretaria de Atenção à Saúde. Departamento de Atenção Básica. Vigilância em Saúde. Brasília; 2008.

7. Oliveira MHP, Romanelli G. Os efeitos da hanseníase em homens e mulheres: um estudo de gênero. Cad Saúde Pública. 1998; 1: 51-60.

8. Brasil. Ministério da Saúde. Secretarias de Políticas da Saúde. Departamento de Atenção Básica. Coordenação de Investigação. Hanseníase. Brasilia; 2000.

9. Pedrazzani ES, Toyoda CY, Brognhara MA, Maluf SA. Controle dos comunicantes: sua interferência na situação epidemiológica da hanseníase. Hansen. Int. 1986; 11: 44-54.

10. Pinto Neto JM. Características epidemiológicas dos comunicantes de hanseníase que desenvolveram a doença, notificados no C.S./ de Fernandópolis (1993 a 1997). [Dissertação]. Ribeirão Preto: Escola de Enfermagem de Ribeirão Preto, Universidade de São Paulo; 1999.

11. Leite KKC, Costa JML, Barral A, Caldas AJM, Corrêa RGCF, Aquino DMC. Perfil epidemiológico dos contatos de casos de hanseníase em área hiperendêmica na Amazônia do Maranhão. Cad Saúde Coletiva. 2009; 1: 235-249.

12. Ferreira IN. Busca ativa de hanseníase na população escolar e distribuição espacial da endemia no município de Paracatu - MG (2004 a 2006). [Dissertação]. Brasília: Faculdade de Saúde, Universidade de Brasília; 2008. 